\title{
Weighted performance based handover parameter optimization in LTE
}

\author{
Thomas Jansen*, Irina Balan ${ }^{\dagger}$, Szymon Stefanski $^{\ddagger}$ Ingrid Moerman $^{\S}$, Thomas Kürner ${ }^{\llbracket}$ \\ * Technische Universität Braunschweig, Braunschweig, Germany, jansen@ifn.ing.tu-bs.de \\ † Interdisciplinary Institute for Broadband Technology, Ghent, Belgium, irina.balan@intec.ugent.be \\ $\ddagger$ Nokia Siemens Networks, Wroclaw, Poland, szymon.stefanski@nsn.com \\ $\S$ Interdisciplinary Institute for Broadband Technology, Ghent, Belgium, ingrid.moerman@intec.ugent.be \\ 『 Technische Universität Braunschweig, Braunschweig, Germany, kuerner@ifn.ing.tu-bs.de
}

\begin{abstract}
Handover parameter optimization is a selfoptimizing network (SON) use case that promises significant performance improvement of the radio network. The basic idea is to adapt the handover control parameters, hysteresis and time-to-trigger, to the individual cell situation, in terms of e.g. the building density, cell environment and degree of user mobility. The aim is to reduce the number of handover failures, ping-pong handovers and radio link failures. We propose an handover parameter optimization algorithm that tunes the hysteresis and time-to-trigger in iterative steps and show the system performance improvement with it in both a realistic and a hexagonal simulation scenario.
\end{abstract}

\section{INTRODUCTION}

Handover is one of the key procedures for ensuring that the users can move freely through the network while staying connected and being offered appropriate service quality. As LTE supports only hard handover (HO), it is vital that this procedure happens as timely and seamlessly as possible. To assure a good handover performance in the network it will sometimes be necessary to alter the handover parameters on a cell basis to account for regional differences between the cells which influence the characteristics of the cell transitions.

This paper continues the work that was already presented in [6]. In this paper we present a new algorithmic approach that is based on the weighted sum of the main handover performance indicators (HPIs) and shows promises good performance across different scenarios and simulation environments. Also, by only needing four input metrics, the new algorithm can easily be adopted and incorporated by multiple vendors/operators. In addition, operators can customize the algorithm by changing the weighting parameters of the individual HPIs to best suit their needs.

The rest of the paper is structured as follows. Section II offers an overview of the input measurements, the control parameters and the assessment metrics used by the algorithm. The algorithm is described in Section III. Section IV presents the scenarios the algorithm was tested in and the results of these simulations are given in Section V. Finally, we draw conclusions and outline future work in Section VI.

The work has been carried out in the EU FP7 SOCRATES project [1] [2].

\section{Metrics \& CONTROL PARAMETERS}

The metrics that are used in the handover parameter optimization (HPO) algorithm are subdivided in system metrics, control parameters and assessment metrics.

The reference signal received power (RSRP) and signalto-interference and noise ratio (SINR) are system metrics. They are used to select the connected cell, possible handover candidates and determine when a radio link failure (RLF) has occurred.

A handover is initiated when the following condition is met: the RSRP of a neighboring cell is greater than the RSRP of the serving cell plus the hysteresis value for at least for the time specified in the time-to-trigger parameter. Hence, these two parameters will be tuned by the HPO algorithm.

- Hysteresis - The considered hysteresis (Hys) values lie between 0 and $10 \mathrm{~dB}$ with steps of $0.5 \mathrm{~dB}$, resulting in 21 considered hysteresis values.

- Time-to-Trigger - The time-to-trigger (TTT) values for LTE networks are specified by 3GPP (see [3] section

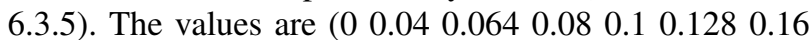
$\left.\begin{array}{lllllllll}0.256 & 0.32 & 0.48 & 0.512 & 0.64 & 1.024 & 1.280 & 2.560 & 5.120\end{array}\right)$ in

[s]. These 16 values are the considered TTT values.

In the remainder of this paper, an unique combination of Hysteresis and TTT values will be referred to as handover operating point (HOP).

The assessment metrics are used as measurements during the optimization process and as performance indicators for the optimization algorithm evaluation. Four metrics are used by the proposed handover optimization algorithm: the three HPIs (the handover failure ratio $\left(H P I_{H O F}\right)$, the ping-pong handover ratio $\left(H P I_{H P P}\right)$ and the radio link failure (RLF) ratio $\left.\left(H P I_{R L F}\right)\right)$ and a fourth one, computed as a weighted sum of the first three.

This fourth assessment metric is called HP (Handover performance) and is an operator policy based weighted sum of the three metrics described above. Hence the HP allows to analyze the handover performance of the network in a single value. The HP is calculated according to the following formula:

$$
H P=w_{1} H P I_{R L F}+w_{2} H P I_{H O F}+w_{3} H P I_{H O P P}
$$


where $w_{i}$ are weights given to each performance indicator.

The values for these weights are a direct translation of the operator policy. The operator of a mobile network can thus influence the performance of the handover algorithm by manipulating the weighting parameters. A combination of $\left[w_{1}=1, w_{2}=0.5, w_{3}=2\right]$, for example, gives priority to the reduction of RLFs, while HO failures are to be avoided but of less importance and ping-pong handovers are allowed to increase as inevitable side effect of the RLF reduction.

More details on how the statistics and parameters in this section are calculated can be found in previous work [6].

\section{HANDOVER OPTIMIZATION ALGORITHM}

The weighted performance based handover optimization algorithm (WPHPO) changes the handover operating points of individual cells in order to increase the handover performance. The current handover performance of a cell is determined by the weighted sum of the three HPIs according to equation 1.

The range of allowed HOPs is limited to a subset lying on a diagonal line in the handover operating space. This limitation is necessary since the optimization algorithm needs to be able to either increase or decrease the HOP and therefore an ordering of the allowed HOPs is required. The drawback of this limited set of handover operating points is that it cannot be guaranteed that the best handover operating point for the given weighting parameters will be found by the optimization algorithm.

However, it has been analyzed in the controllability and observability studies in [6] that the performance difference between handover operating points lying in a ditch in the handover operating space can be neglected. Every diagonal line in the handover operating space crosses this ditch and hence an optimization into this ditch is possible. The handover operating space is defined by the standardized time-to-trigger and hysteresis values that have been introduced before. Figure 1 shows examples of the subsets of allowed handover operating points taken into account for the optimization. The terms and conditions for choosing a diagonal line of allowed handover operating points are the following:

- All time-to-trigger values have to be considered for the optimization.

- At least 16 different hysteresis values have to be considered.

- The allowed handover operating points have to lie on a diagonal line in the handover operating space following a straight line or a step-function as shown in Figure 1.

In the case the allowed handover operating points lie on a step-function in the handover operating space the optimization speed decreased since the optimization algorithm only changes the TTT or Hys value at a time. However the granularity of adjusting the handover parameters to the current network situation increases in this case.

Figure 2 presents a flowchart of the WPHPO algorithm that will be described in detail in this section.

Before the optimization algorithm is initiated all cells in the network have to select an allowed handover operating point.

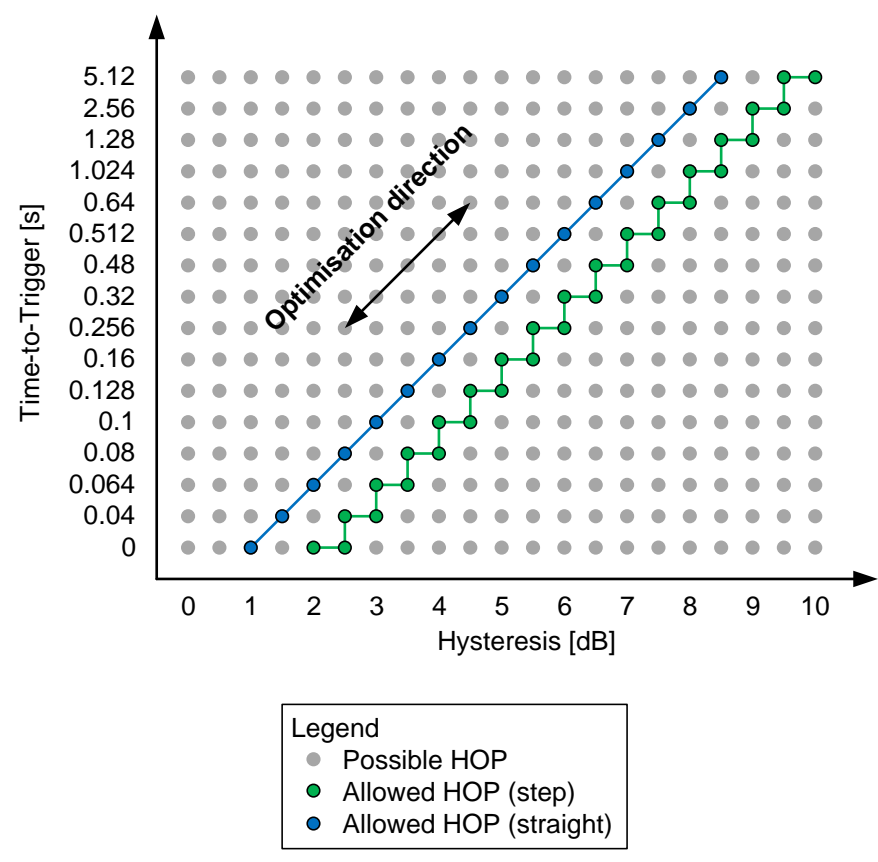

Fig. 1. Allowed handover operating points

The operating point with a hysteresis value of $6 \mathrm{~dB}$ and a timeto-trigger of $0.32 \mathrm{~s}$ turned out to be a good starting point for the optimization since it showed good handover performance for the cells in most of the considered scenarios. The starting HOP does not influence the optimization result in the long run but it may influence the adaptation speed in the beginning of the simulation. For HOPs with very small or very high values the optimization algorithm may need quite a number of optimization steps before a good handover performance is reached.

In addition the initial optimization direction has to be selected for all cells. The optimization direction is continuously switched if the optimization algorithms experiences worse handover performance compared to the last HOP in the current configuration. Since there is no last HOP in the beginning of the optimization the initial optimization direction has to be defined. It will not influence the optimization capability of the algorithm in the long-run but can influence the performance of the algorithm in the starting phase.

In every time step the handover performance indicators are collected. The optimization is carried out in fixed optimization intervals as shown in Figure 3 only. In these intervals the handover performance HP is calculated from the HPI values including the weighting parameters that have been introduced before. The HPI window defines the time that is considered for the calculation of the HPIs. This window should be smaller than the optimization instance to assure that the HPIs are not influenced by earlier HO parameter settings. If the HP is equal to zero no optimization is needed since the HPIs are all equal to zero as well. If the HP is not equal to zero the handover performance of the current handover operating point is compared to the performance of the last handover operating 


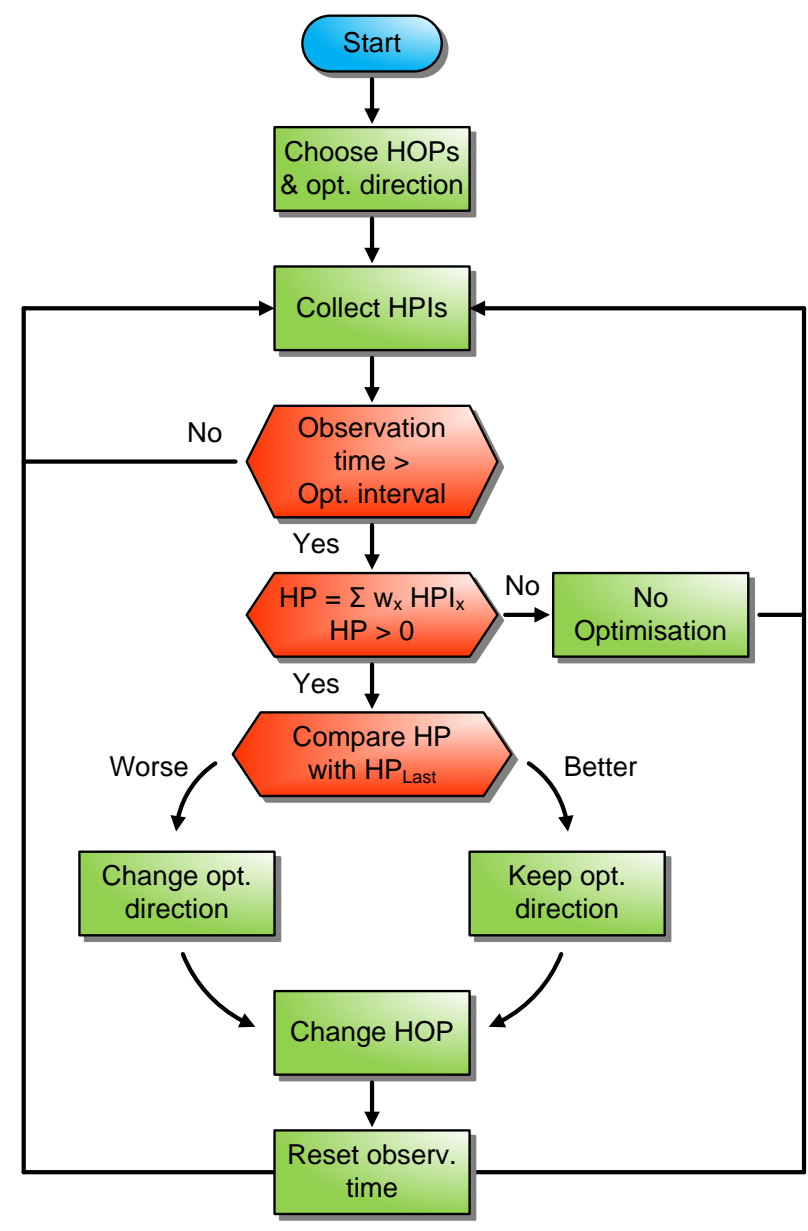

Fig. 2. Flowchart of the WPHPO algorithm

point of the cell.

During initialization, the HOP of all the eNBs in the scenario is set to the same value. Subsequently, each eNB will change the current HOP individually, based on the observed cell performance. There is no direct relationship between the HOP settings/changes of neighboring eNBs (group behavior or alignment). However, the diagonal on which the optimization will be carried out, is dependent on the initial HOP setting, since this diagonal has to pass through this HOP. For very extreme or inappropriate initial HOP setting, the algorithm may perform sub-optimally since the absolute minimum might be impossible to reach and a local minimum will be achieved instead.

If the performance of the current handover operating point is worse the optimization direction is switched and a new handover operating point in this direction is chosen. If the performance of the current handover operating point is better, a new handover operating point is chosen in the current optimization direction. The HOPs can be found by going up and down the allowed handover operating points as shown in 1. Finally the HP of the last handover operating point and the handover optimization direction are saved since they are needed for the next optimization decision.

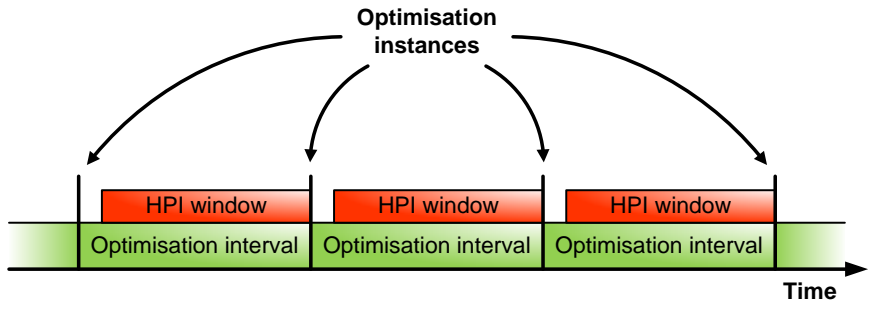

Fig. 3. Optimization instances of the WPHPO algorithm

The decision to use the HP as the input parameter for this algorithm, in opposite to base the optimization on the single HPI values on their own, has two advantages:

- The HP offers a more complete view on the network performance. Since the three HPIs are closely related, certain compromises must be made in order to improve the overall handover performance. For instance, a HOP setting that minimizes RLFs will most probably increase the number of ping-pong handovers.

- The weights used for calculating the HP are a direct translation of the operator policy. Hence this approach enables influence of the operator on the optimization goal. The operator can adapt the weights of the HP (or the their relative value to each other) to control the resulting handover performance.

As mentioned before, the weights we use in this algorithm are a direct translation of the operator policy. Although any combination of weights is possible when calculating the HP as shown in equation 1, not all such combinations actually make sense. If, for example, all weights are set to the same value (all three statistics are given equal importance), this may result in suboptimal network performance (the number of RLF will be leveled with the that of the ping-pongs and the HO failures although the latter happen very seldom). If the weight of the RLF is equal to the one of the ping-pongs, this for sure will introduce oscillation of the HOP since once the RLF ratio decreases, the ping-pong ratio increases. Also, extreme values for one weight compared to the other two, will probably reduce that HPI but will increase one of the other two well beyond maximum threshold (e.g. $R L F \geq 50 \%$ or $P P \geq 70 \%$ ). So, as input for the WPHPO, the operator must set some realistic targets which will be reflected in the values of the weights. The rest of the paper shows results that use the following weight combination: [ $\left.w_{1}=1, w_{2}=0.5, w_{3}=2\right]$ that reflect an operator that is mainly interested in reducing the number of RLFs.

A similar compromise must be achieved for setting an optimization interval for the algorithm as well. A small optimization interval would be less reliable, providing less statistical relevance, and thus may trigger optimization actions based on uncertain measurements (i.e. one extra radio link failure of only a few initiated handovers can result in a much higher radio link failure ratio). On the other hand, even if unnecessary changes are made due to insufficient information, the optimization will be faster and it will affect the users for 
a shorter time if a small optimization interval is chosen. The HOP will undergo more adjustments in the same amount of time, thus helping faster convergence. Simulation times are also taken into account (if the optimization interval is large, the total simulation time will have to be longer in order to permit a certain number of changes). We focus on an the optimization interval of $120 \mathrm{~s}$ in this paper since this assures meaningful handover statistics and limited simulation time.

\section{Simulation SCEnARIOS}

In order to reflect more realistic relations in the simulated scenario, a non-regular network layout has been chosen for the network simulation ( see [7] [8] for more details). Figure 4 shows a network layout of 12 sites, 3 sectors per site resulting in 36 cells with different inter site distance (ISD) between them. The grid of different cell sizes and irregular shapes creates a diversified environment with interference limited areas (small size cells where the interference is the limiting factor) which are in neighborhood of coverage limited areas (large cells). In such a miscellaneous system, 14 users per cell have been randomly deployed and one group of 50 concentrated users moves with the constant velocity of $30 \mathrm{~km} / \mathrm{h}$ in a random direction. More details on the simulation assumptions can be found in the 3GPP definitions [9].

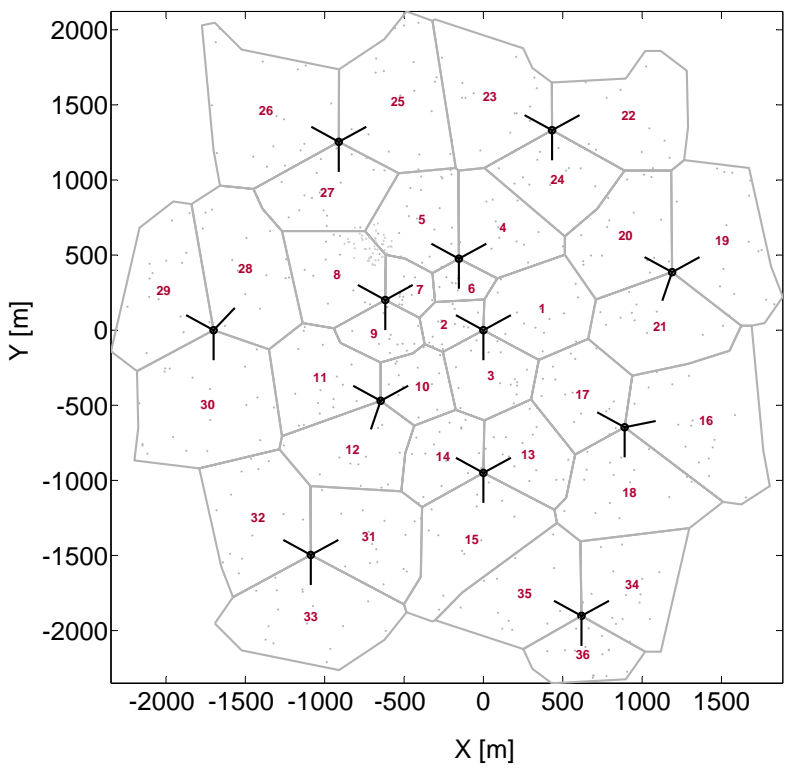

Fig. 4. The springwald scenario

Handover optimization is more difficult in irregular networks since the irregular shape results in both small and large overlap areas, i.e. the areas between neighboring cells where a connection to either of the two or even more cells is possible. The optimal handover parameter settings depend on the size of these areas and thus an optimization of the handover parameters is beneficial.

In addition to the non-regular network layout the realistic SOCRATES scenario has been used for the system simulations. Figure 6 shows a small section of the SOCRATES scenario. All users are moving with a speed of 0 to $50 \mathrm{~km} / \mathrm{h}$ on the streets in the shown area with a size of $1.5 \mathrm{~km} *$ $1.5 \mathrm{~km}$. The network is based on the antenna positions of an operator and the path losses to the users are calculated using a ray-tracer. More details on the realistic SOCRATES reference scenario can be found in [4] and [5].

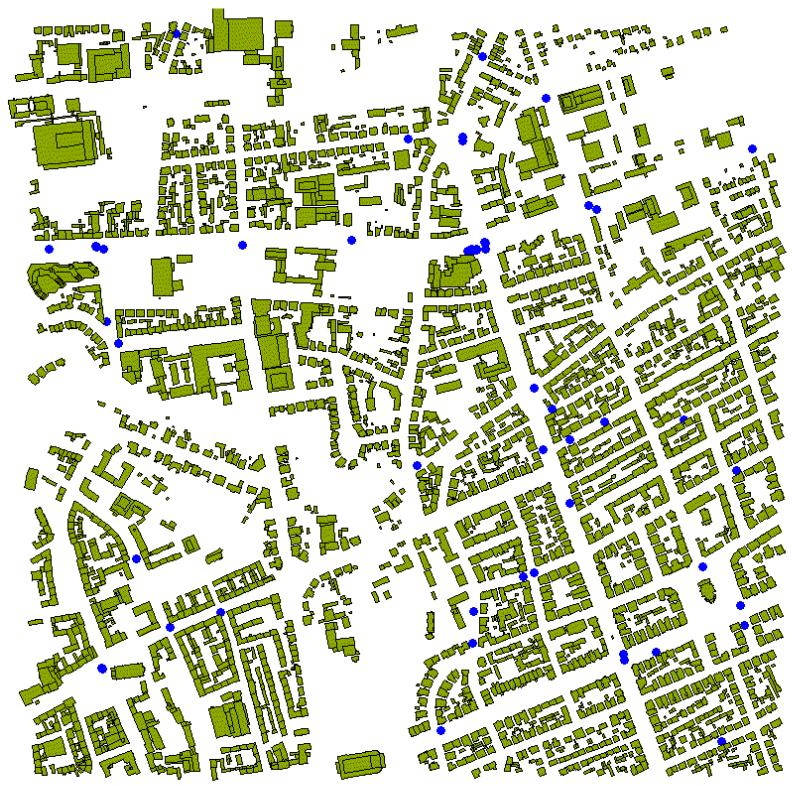

Fig. 5. The SOCRATES reference scenario

\section{Simulation Results}

The simulation results using the non-regular network layout are shown in Figure 6 and provide an evaluation on the performance gain achieved by the WPHPO algorithm. In the reference case the WPHPO algorithm is disabled, the hysteresis and TTT parameters are fixed in each cell and adjusted respectively to $4.5 \mathrm{~dB}$ and $300 \mathrm{~ms}$. In the optimization case the WPHPO algorithm reduces the number of radio link and handover failures significantly at the expense of a higher ping-pong handover ratio which is a desired effect according to the weighting parameter settings.

At the beginning of the simulation (before 120s) no difference between the reference and optimization case are visible in all charts because of the HPIs averaging window size of 120 s. Hence the first optimization actions are triggered after 120s. During the first optimization steps (approximately before 400s) much higher RLF ratios are observed in the case the WPHPO algorithm is activated. This effect can be explained by the initial optimization direction that has to be selected before the simulation. After this period the WPHPO algorithm switches the optimization direction and the curves of the RLF ratio as well as HP show significant performance gain until the end of the simulation.

These simulation results show that the WPHPO is able to improve the handover performance of a network significantly. The selected starting HOPs showed a good overall handover performance without optimization in the network already. 
Nevertheless the WPHPO still improves the HO performance after some time.
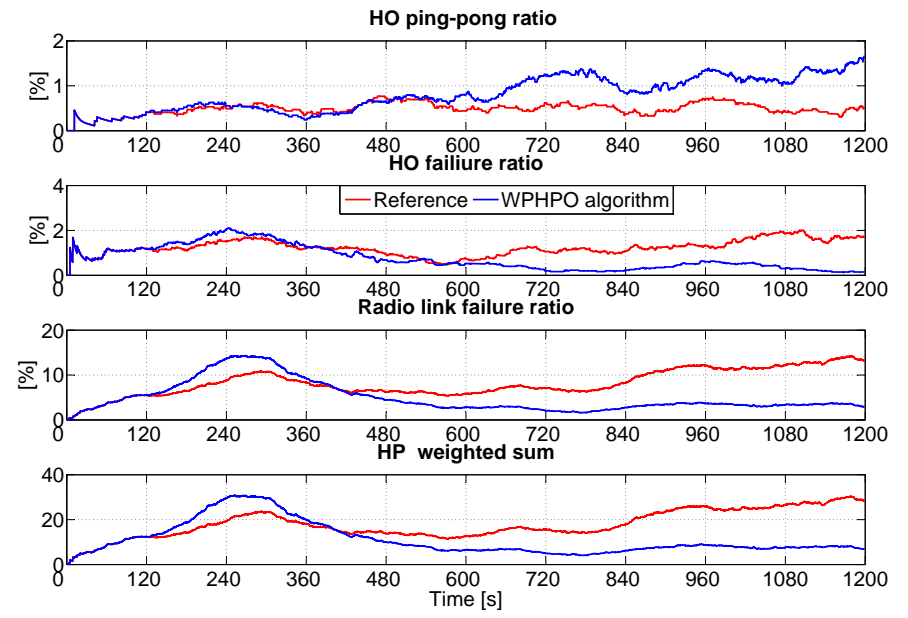

Fig. 6. Handover performance in the springwald scenario

The results of the system simulations using the realistic SOCRATES scenario are depicted in Figure 7. For these simulations 50 mobile user were considered that traveled along the streets in the scenario. The difference to the simulations in the non-regular scenario is that the WPHPO algorithm starts the optimization with a non-optimal HOP setting in the network. All cells use the HOP with a hysteresis of $7.5 \mathrm{~dB}$ and a TTT of $1.024 \mathrm{~s}$ in the beginning of the simulation. The shown reference simulation was done with a fixed HOP using a hysteresis of $5 \mathrm{~dB}$ and a TTT of $320 \mathrm{~ms}$ in all cells. This HOP shows the best performance of all fixed HOP settings that have been considered.

In the beginning of the simulation the RLF ratio of the WPHPO algorithm is worse than the reference case but no handover failures and ping-pong handovers are observed. According to the controllability studies, that have been mentioned before, this is the expected handover performance for higher HOPs. After 450 seconds simulation time the RLF ratio shows a high peak. Again this peak is caused by the wrong optimization direction that has randomly been chosen. After the direction has been altered the WPHPO algorithm continuously improves the $\mathrm{HO}$ performance in the network.

The simulation results show that the WPHPO algorithm improves the HO performance even if sub-optimal HOP settings are chosen as starting condition. The optimization actions constantly improve the network performance and keep it low after a good $\mathrm{HO}$ performance is reached.

\section{CONCLUSION \& OUtLOOK}

The proposed WPHPO algorithm has shown a high capability in HO performance improvement. Different simulation scenarios and $\mathrm{HO}$ parameter settings have been considered to show the optimization capabilities of the WPHPO algorithm. The advantage of the proposed solution is that the weighted sum allows to identify better handover performance accounting for different operator policies that can be translated to the
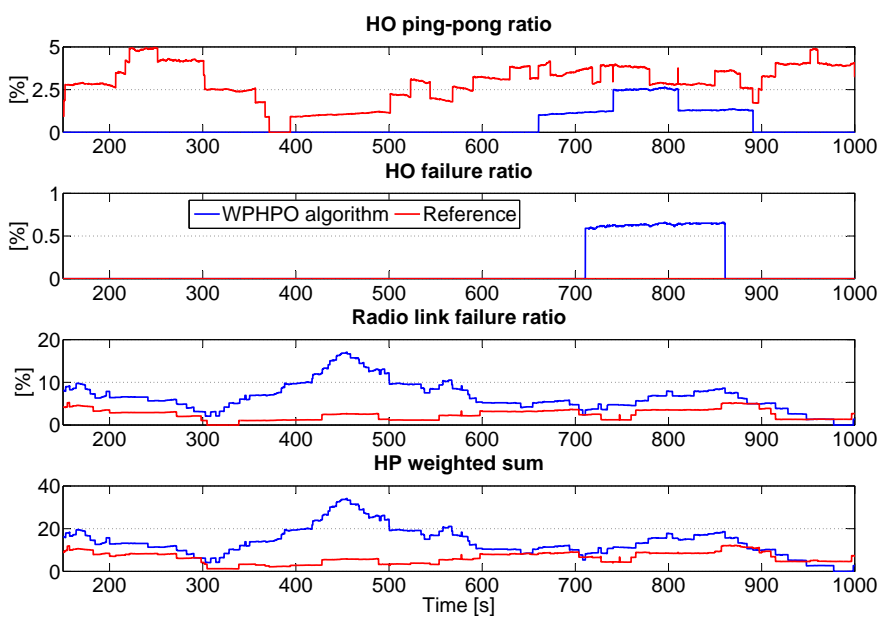

Fig. 7. Handover performance in the realistic SOCRATES scenario

algorithm settings. Hence the algorithm can be used for individual goals of different network operators.

Moreover the optimization speed of the WPHPO algorithm can be influences by changing the optimization interval on the one hand or the considered HOPs on the other hand. Thus the WPHPO algorithms can be tuned in many ways to serve the needs of different operators.

However to analyze the behavior of the algorithm and suggest different settings for certain environments and optimization targets the algorithms has to be further evaluated in different scenarios. The influence of the optimization interval and the selected diagonal in the optimization space plays a key role in these investigations.

\section{REFERENCES}

[1] SOCRATES, Self-optimisation and self-configuration in wireless networks, European Research Project, http://www.fp7-socrates.eu.

[2] M. Amirijoo, R. Litjens, K. Spaey, M. Döttling, T. Jansen, N. Scully, and $\mathrm{U}$. Türke, Use Cases, Requirements and Assessment Criteria for Future Self-Organising Radio Access Networks, 3rd International Workshop on Self-Organizing Systems, IWSOS 08, Vienna, Austria, December 2008.

[3] 3GPP, 3rd Generation Partnership Project; Technical Specification Group Radio Access Network; Evolved Universal Terrestrial Radio Access (EUTRA) Radio Resource Control (RRC); Protocol specification (Release 9), Technical Report TR 36.331 v9.1.0, available at http://www.3gpp.org.

[4] N. Scully, J. Turk, R. Litjens, U. Türke, M. Amirijoo, T. Jansen and L. C. Schmelz Review of use cases and framework II, Deliverable 2.6 EU-Project SOCRATES, December 2009.

[5] H. Schumacher, M. Schack and T. Kürner, Coupling of Simulators for the Investigation of Car-to-X Communication Aspects, 7th COST2100 Management Committee Meeting, TD(09)773, Braunschweig, Germany, February 2009.

[6] T. Jansen, I. Balan, J. Turk, I. Moerman and T. Kürner, Handover parameter optimization in LTE self-organizing networks, IEEE 72nd Vehicular Technology Conference (VTC2010-Fall), Ottawa, Canada, September 2010.

[7] J. Turkka and A. Lobinger, Non-regular Layout for Cellular Network System Simulations, PIMRC 2010, Istanbul, Turkey, September 2010.

[8] A. Lobinger, S. Stefanski, T. Jansen and I. Balan, Load Balancing in Downlink LTE Self-Optimizing Networks, IEEE 71st Vehicular Technology Conference (VTC2010-Spring), Taipei, Taiwan, May 2010.

[9] 3GPP, Physical Layer Aspects for evolved Universal Terrestrial Radio Access (E-UTRAN), Technical Report TR 25.814, available at http://www.3gpp.org. 